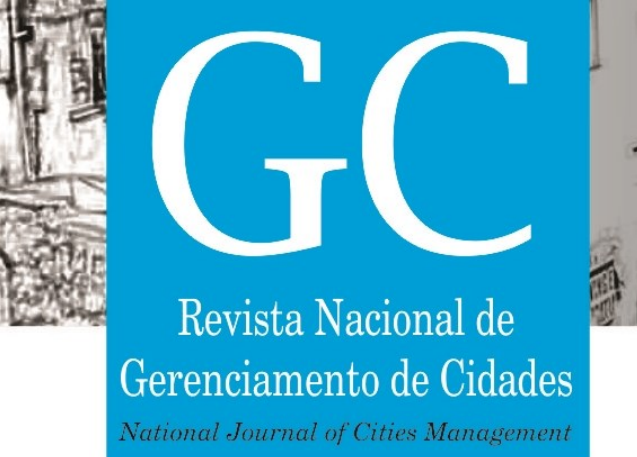

ISSN 2318-8472

v. 04, n. 25,2016

\title{
Análise de implantação de Abrigo Emergencial em Joinville
}

Emergency Shelter implementation analysis in Joinville

Análisis de la implementación de alojamiento de emergencia en Joinville

Nathalia Louise Giordani

Graduada em Engenharia de Infraestrutura, UFSC, Brasil nathilgiordani@gmail.com

Andréa Holz Pfützenreuter

Professor Doutor, UFSC, Brasil andrea.hp@ufsc.br 


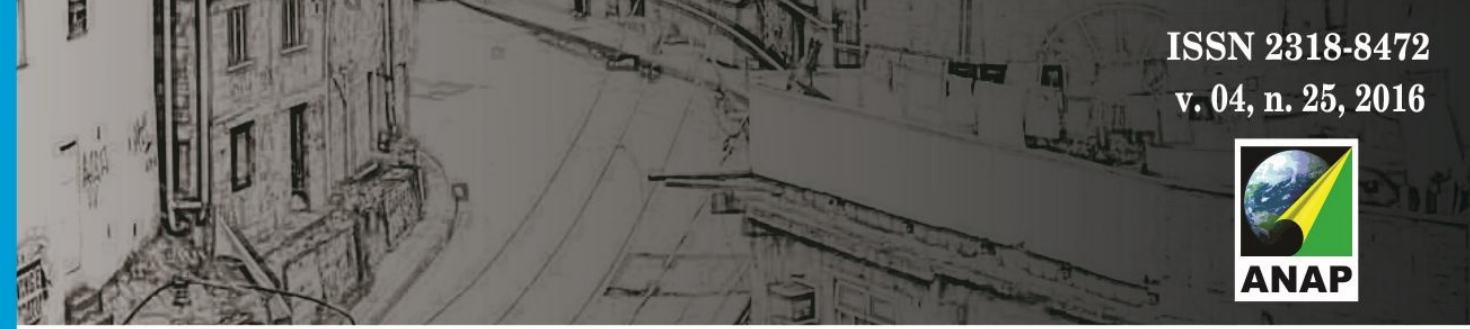

Gerenciamento de Cidades

\section{RESUMO}

O crescente número de vítimas de desastres naturais é uma preocupação constante das políticas públicas. A importância de alcançar um sistema construtivo de rápido fornecimento, eficiente, baixo custo, exequível e com qualidade final confortável aos usuários, são características relevantes à implantação de um Abrigo Emergencial Temporário para pessoas que tiveram seus lares afetados por conta de desastres naturais, estando em condições de moradia debilitadas ou mesmo sem ela. $\mathrm{O}$ objetivo deste trabalho é analisar o projeto de um abrigo emergencial vencedor do concurso internacional promovido pela Autodesk em 2015, estudando possíveis trocas de materiais e do sistema construtivo, para viabilizar economicamente sua reprodução em escala; entender a exeqüibilidade do projeto realizado por terceiros e de indicar a eficiência ao fornecimento das partes. Por meio de pesquisa de precificação de mercado dos materiais e análise da viabilidade logística de fornecimento do sistema de Kits para os Abrigos Temporários mas com a premissa de manter as linhas arquitetônicas projetuais nas modificações de materiais e sistemas está comprovado que o abrigo torna-se viável economicamente.

PALAVRAS-CHAVE: Abrigo emergencial. Viabilidade. Sistema construtivo.

\section{ANSTRACT}

The growing number of victims of natural disasters is a constant concern of public policy. The importance of achieving a constructive system of fast delivery, efficient, low cost, practical and comfortable quality end users, are relevant features to the implementation of an Emergency Shelter Temporary for people who have had their homes affected due to natural disasters, being in debilitated housing conditions or even without it. The objective of this study is to analyze the design of an emergency winner under international competition sponsored by Autodesk in 2015 , studying possible exchange of material and construction system for economically viable reproduction scale; understand the feasibility of the project carried out by others and to indicate the efficiency of the supply of parts. Through market of materials and analysis of the feasibility logistics supply Kits system pricing research for Temporary Shelters but with the premise of maintaining the architectural lines projective in changes in materials and systems is proven that the shelter becomes feasible economically.

KEYWORDS: Emergency shelter. Viability. Construction system.

\section{RESUMEN}

El creciente número de víctimas de desastres naturales es una preocupación constante de la política pública. La importancia de lograr un sistema constructivo de entrega rápida, eficiente y de bajo costo, los usuarios finales funcional y confortable calidad, son las características relevantes para la implementación de un refugio de emergencia temporal para las personas que han perdido sus casas afectadas por desastres naturales, estando en debilitado las condiciones de vivienda o incluso sin ella. El objetivo de este estudio es analizar el diseño de un ganador de emergencia en condiciones de competencia internacional patrocinada por Autodesk en 2015, el estudio de posible intercambio de sistema de construcción de material y para la escala de reproducción económicamente viable; entender la viabilidad del proyecto llevado a cabo por otros y para indicar la eficiencia del suministro de piezas. A través del mercado de materiales y análisis de la investigación de equipos de suministro de logística de factibilidad sistema de precios para los refugios temporales, pero con la premisa de mantener las líneas arquitectónicas proyectiva en cambios en los materiales y sistemas se ha demostrado que el refugio se convierte en viable económicamente.

PALABRAS CLAVE: Refugio de emergência. Factibilidad. Sistemas de Construcción 
Este artigo é resultado da análise da viabilidade de implantação de projeto arquitetônico desenvolvido por estudantes do curso de Arquitetura e Urbanismo, de um Abrigo Temporário Emergencial para Joinville, vencedor do concurso internacional promovido pela Autodesk. As possíveis trocas de materiais e do sistema construtivo, além de entender sua exequibilidade e indicar eficiência no fornecimento das partes são diretrizes importantes a fim de possibilitar sua reprodução em escala.

\section{DEFINIÇÕES E CARACTERÍSTICAS PROJETUAIS}

O projeto trata de um abrigo preparado para situações de emergência, por meio do desenvolvimento de um kit pré-fabricado visando uma solução de construção em menor tempo de aplicação e reutilização.

seriam um total 124 abrigos, com um total novamente de 372 pessoas, e 124 famílias.

A proposta do abrigo emergencial para Joinville (AEJ) se adapta em uma vaga de estacionamento de $2,4 \mathrm{~m} \times 5 \mathrm{~m}$, havendo a possibilidade de ampliação de um quarto, na vaga adjacente. $O$ local escolhido para aplicação foi o estacionamento adjacente ao bloco de ensino em razão da amplitude e por proporcionar replicação da instalação do abrigos, sendo uma alternativa de implantação para situações emergenciais futuras em diversos contextos, uma vez que esses pátios são geralmente encontrados em todas as regiões das cidades.

O projeto atende o conceito de modulação, que pode ser definido como uma medida adotada que regula a proporção de diversas partes de uma construção, de modo que diferentes elementos resultem em um todo harmônico. A proposta abrange duas soluções de construção: o módulo base (figura 1 e 2,) que conta com área de $12 \mathrm{~m}^{2}$ e com capacidade de abrigar 4 pessoas, e o módulo base com ampliação (figura 3 e 4), com 21,12 $\mathrm{m}^{2}$, e possui a capacidade de abrigar até 6 pessoas.

Foi realizada pesquisa de precificação de mercado dos materiais, e análise da viabilidade logística de fornecimento do sistema de Kits para os Abrigos Temporários. A proposição do projeto são conceitos alinhados no sistema flat-pack, ou seja, uma montagem otimizada e padronizada, em razão da modulação, o que torna possível a montagem de kits pré-fabricados, para fornecimento e posterior utilização. 
Tabela 1 - Custos dos materiais utilizados para o AEJ

\begin{tabular}{|c|c|c|c|c|c|c|}
\hline \multirow{2}{*}{\multicolumn{2}{|c|}{ ITEM }} & \multirow[b]{2}{*}{ TIPO DE MATERIAL } & \multicolumn{4}{|c|}{ CUSTO DO MATERIAL } \\
\hline & & & $\begin{array}{c}\text { Custo } \\
\text { Unitário }\end{array}$ & $\begin{array}{l}\text { MÓDULO } \\
\text { BASE }\end{array}$ & AMPLIAÇÃO & $\begin{array}{c}\text { MÓD BASE + } \\
\text { AMP }\end{array}$ \\
\hline \multicolumn{7}{|c|}{ 1. INFRAESTRUTURA } \\
\hline 1.1 & Fundação & Pallets PET reciclado & 150,00 & 3000,00 & 2700,00 & 5700,00 \\
\hline 2.2 & Piso & Piso Compensado Naval ( $25 \mathrm{~mm})$ & 189,00 & 614,25 & 448,8 & 1063,13 \\
\hline \multicolumn{7}{|c|}{ 2. FECHAMENTO } \\
\hline \multirow{2}{*}{3.1} & \multirow{2}{*}{$\begin{array}{l}\text { Fechamento } \\
\text { Externo }\end{array}$} & Placas de PVC & 274,00 & 11546,36 & 8915,96 & 20462,32 \\
\hline & & Placa de Policarbonato Compacto & 1900,00 & 2042,50 & 266,00 & 2308,50 \\
\hline 3.2 & $\begin{array}{l}\text { Fechamento } \\
\text { Interno }\end{array}$ & Compensado Naval (18mm) & 135,00 & 1551,35 & 1214,62 & 2765,97 \\
\hline \multicolumn{7}{|c|}{ 3. ESTRUTURA } \\
\hline 3.1 & Viga & Madeira Plástica para travamento & 25,39 & 303,66 & 179,00 & 482,66 \\
\hline 3.2 & Esquadrias & Madeira Plástica nas esquadrias & 22,60 & 787,61 & 224,19 & 1011,80 \\
\hline 3.3 & $\begin{array}{l}\text { Estrutura } \\
\text { interna de } \\
\text { parede }\end{array}$ & Wood frame & 97,61 & 4113,29 & 3176,23 & 7289,51 \\
\hline 3.4 & $\begin{array}{l}\text { Fixação da } \\
\text { estrutura }\end{array}$ & Parafuso passante c/ Porca Borboleta & 11,50 & 299,00 & 207,00 & 506,00 \\
\hline & & & TOTAIS & $24.258,02$ & $17.331,87$ & $41.589,89$ \\
\hline
\end{tabular}

Conforme exposto acima, os valores encontrados para o Módulo Base foi $\mathrm{R} \$ 24.258,02$ e para o Módulo Base mais Ampliação de R\$ 41.589,89. Os valores calculados são apenas dos materiais necessários para a contrução de uma unidade de cada, sem incluir nesse preço, os custos adicionais de transporte, benefícios e despesas indiretas (BDI), impostos, mão de obra para montagem, entre outros.

Como mencionado anteriormente, existem duas situações de implantação dos módulos no estacionamento, nomeadas aqui de: situação 1 (com apenas módulos base) e a situação 2 (com módulos base e módulos base com ampliação). Com os custos definidos na tabela 1 , apresenta-se o valor total dos módulos na tabela 2 .

\begin{tabular}{|c|c|c|c|c|c|c|c|c|}
\hline & \multirow{2}{*}{$\begin{array}{l}\text { Capac. } \\
\text { (pessoas) }\end{array}$} & \multirow{2}{*}{$\begin{array}{c}\text { Valor } \\
\text { Unitário (R\$) }\end{array}$} & \multicolumn{3}{|c|}{ SITUAÇÃO 1} & \multicolumn{3}{|c|}{ SITUAÇÃO 2} \\
\hline & & & $\begin{array}{l}\text { Quant. } \\
\text { (mód) }\end{array}$ & TOTAL (R\$) & $\begin{array}{c}\text { Total } \\
\text { (pessoas) }\end{array}$ & $\begin{array}{l}\text { Quant. } \\
\text { (mód) }\end{array}$ & TOTAL (R\$) & $\begin{array}{c}\text { Total } \\
\text { (pessoas) }\end{array}$ \\
\hline MÓDULO BASE & 3 & $24.258,02$ & 124 & $3.007 .994,38$ & 372 & 16 & $388.128,307$ & 48 \\
\hline \multirow[t]{3}{*}{$\begin{array}{l}\text { MÓDULO BASE + } \\
\text { AMPLIAÇÃO }\end{array}$} & \multirow[t]{3}{*}{6} & $41.589,89$ & - & - & - & 54 & $2.245 .854,147$ & 324 \\
\hline & & TOTAIS & 124 & $3.007 .994,38$ & 372 & 70 & $2.633 .982,45$ & 372 \\
\hline & & FAMÍLIAS & & & 124 & & & 70 \\
\hline
\end{tabular}


Assim, observa-se que as duas situações apresentadas resultaram a mesma quantidade de pessoas, no entanto, a situação 2 apontou um custo de aproximadamente $\mathrm{R} \$ 375$ mil a menos que a situação 1 . Com a diretriz da pesquisa para a opção econômica, essa seria a situação mais apropriada para alocação de famílias no estacionamento.

\section{ANÁLISE LOGÍSTICA PARA O TRANSPORTE}

A proposta de anteprojeto desenvolvido por Alano e Tomaz (2015), apresenta o encaixe da estrutura, entretanto não consta a informação dos mesmos desmontados e suas partes especificadas, muito menos estudos de viabilidade de transporte e logística, tanto na parte de embalagem dos kits, como para transportá-los ao consumidor final. Desta forma, o estudo do processo de montagem e desmontagem com uma análise logística de transporte dos módulos se faz necessário para verificação da viabilidade de concretização material dos kits.

As unidades são formadas por um kit de placas de fechamento, onde a construção do módulo é simplificada por arcos estruturais já montados de forma pré-fabricada, com encaixe machofêmea, e mobilização com parafusos borboleta.

Analisadas as possíveis probabilidades de montagem dos kits com o objetivo de adquirir as menores dimensões possíveis, testou-se as possibilidades de empilhamento dos kits de acordo com o sistema construtivo, utilizando arcos agrupados com sistema de encaixe (nomeados como KITS 1,2,3, A, B). A concepção da desmontagem desses elementos foi considerada conforme esse sistema proposto. No entanto, como são placas estruturais, que possuem encaixe macho fêmea, foi viabilizado mais um kit, sem considerar essa união dos arcos inicialmente projetado (KITS 4 e C). Abaixo segue a tabela 3, de como ficariam a dimensões desses kits modelados, bem como sua análise de exequibilidade.

Tabela 3 - Análise da Exequibilidade dos Kits

\begin{tabular}{|c|c|c|c|c|c|}
\hline $\begin{array}{c}\text { Possibilidades } \\
\text { de KITS }\end{array}$ & Comprimento $(\mathrm{m})$ & Largura $(\mathrm{m})$ & Altura $(\mathrm{m})$ & Exequibilidade & $\begin{array}{c}\text { Quantidade } \\
\text { por Container }\end{array}$ \\
\hline KIT 1 & 3,45 & 2,40 & 1,42 & Inexequível & - \\
\hline KIT 2 & 3,45 & 2,20 & 1,80 & Exequível & 3 \\
\hline KIT 3 & 3,45 & 1,20 & 2,55 & Exequível & 3 \\
\hline KIT 4 & 3,45 & 2,50 & 1,11 & Inexequível & - \\
\hline KIT A & 3,65 & 2,40 & 1,01 & Inexequível & - \\
\hline KIT B & 3,65 & 1,80 & 1,23 & Exequível & 6 \\
\hline KIT C & 3,65 & 2,10 & 1,06 & Exequível & 6 \\
\hline
\end{tabular}

Considerando para o transporte a utilização de caminhões container de modelo 40 High Cube $(\mathrm{HC})$, que apresenta as dimensões internas: Comprimento $(12,05 \mathrm{~m})$, Largura $(2,347 \mathrm{~m})$ e Altura $(2,695 \mathrm{~m})$. A tabela 3 acima, além de apresentar as dimensões dos kits, é possível observar que os kits 1, 4 e A, se tornam inexequíveis, em razão de sua medida da largura ultrapassar o valor de $2,347 \mathrm{~m}$ do container, tornando-os, inviáveis de utilizar no estudo. Por segundo, os kits 4 e $C$ são as medidas dos módulos que foram empilhados sem considerar os arcos de agrupamento 
utilizados. Mediante o exposto, não foram alcançadas mudanças relevantes e representativas nos kits mencionados, pois o KIT 4 se inviabilizou pela sua largura, e o KIT 6, permaneceu com 6 unidades ajustadas no container.

Desta forma, existindo duas situações para implantação dos módulos no local, onde a situação 2 é a escolha mais econômica, contendo 16 Módulos Base mais 54 Módulos Base com Ampliação, com um total de 70 unidades. Para o transporte do módulo base seriam necessários 6 caminhões, onde um deles transportaria apenas um kit, o que se torna o custo muito elevado, sendo assim exclui-se um caminhão dessa operação. E para o módulo base mais ampliação seriam necessários 9 caminhões, totalizando quatorze caminhões para o transporte. Sendo uma demanda grande de caminhões para um mesmo lugar, percebe-se que o valor de transporte seria alto.

Tendo em vista a quantidade de caminhões e o valor agregado a implantação dos abrigos emergenciais deste valor, justifica-se um estudo de alterações de projeto com enfoque no módulo base, para que se consiga otimizar a quantidade de caminhões, tendo em vista que a quantidade de famílias atendidas também é o mesmo para ambos os módulos.

Sendo assim, sugere-se a investigadas possibilidades de alteração de projeto, tanto para materiais como do sistema construtivo, e propondo novas soluções, para tornar o abrigo exequível, com um melhor custo-benefício e com transporte facilitado.

\section{VIABILIDADE DE ADAPTAÇÕES}

Com o embasamento no quantitativo do AEJ e da análise de viabilidade inicial, surgiriam as propostas de mudanças, tanto em questão de materiais quanto em sistema construtivo, de forma a otimizar o projeto, sem descaracterizae forma a otimizar o projeto, mas sem descaracteriza-lo arquitetonicamente..

Desta forma, as alternativas analisaram o melhoramento de cada parte do sistema, levando em conta os aspectos de custo e troca de materiais, baseados nos critérios de Gibb e Isack (2003), de Segurança, Produtividade, Qualidade Final e Eficiência.

Mediante a análise dos valores dos materiais para a construção dos módulos por meio de pesquisa de preços para o projeto original e também para os novos materiais e sistema construtivo, apresentados na tabela 4. Para esse novo estudo de preços, considerou-se somente o Módulo Base, com o objetivo de concentrar a otimização e padronização. 
Tabela 3 - Quantitativo de materiais e custos, utilizados na nova proposta para o AEJ

\begin{tabular}{|c|c|c|c|c|c|c|c|}
\hline \multirow[b]{2}{*}{ ITEM } & \multirow{2}{*}{ TIPO DE MATERIAL } & \multirow{2}{*}{$\begin{array}{l}\text { DIMENSÃO } \\
(\mathrm{mm})\end{array}$} & \multirow{2}{*}{ UN } & \multirow{2}{*}{$\begin{array}{l}\text { MÓDULO } \\
\text { BASE }\end{array}$} & \multirow{2}{*}{$\begin{array}{l}\text { QTDADE DE } \\
\text { MATERIAL } \\
\begin{array}{c}\text { MÓDULO } \\
\text { BASE }\end{array}\end{array}$} & \multicolumn{2}{|c|}{$\begin{array}{l}\text { CUSTO DO } \\
\text { MATERIAL }\end{array}$} \\
\hline & & & & & & $\begin{array}{l}\text { Custo } \\
\text { Unitário }\end{array}$ & $\begin{array}{l}\text { MÓDULO } \\
\text { BASE }\end{array}$ \\
\hline \multicolumn{8}{|l|}{ 1. INFRAESTRUTURA } \\
\hline \multirow{6}{*}{ Piso } & Pallet de madeira usado & $25 \times 1200 \times 2200$ & $*$ & 20 & 20 & 10,00 & 200,00 \\
\hline & sem pallets & - & - & - & - & - & - \\
\hline & $\begin{array}{l}\text { Deck injetado de } \\
\text { madeira plástica }\end{array}$ & $500 \times 500$ & $\mathrm{~m}^{2}$ & 12 & 48,0 & 81,15 & 3895,20 \\
\hline & Lona resistente (caminhão) & $1400 \times 5000$ & $\mathrm{~m}^{2}$ & 12 & 12,00 & 33,33 & 399,96 \\
\hline & Lona Vinil & 1400(largura) & $\mathrm{m}$ & 12 & 12,00 & 21,42 & 257,04 \\
\hline & $\begin{array}{l}\text { Piso PVC } \\
\text { emborrachado }\end{array}$ & $1000 \times 1000$ & $\mathrm{~m}^{2}$ & 12 & 12,00 & 34,90 & 418,80 \\
\hline \multicolumn{8}{|l|}{ 2. FECHAMENTO } \\
\hline \multirow{4}{*}{$\begin{array}{c}\text { Fechamento } \\
\text { Externo + } \\
\text { Interno }\end{array}$} & Chapa de acrílico transparente & $8 \times 3000 \times 2000$ & $\mathrm{~m}^{2}$ & 6,83 & 1,14 & 2500,00 & 2845,83 \\
\hline & Cortina de PVC transparente & 200(largura) & $\mathrm{m}^{2}$ & 6,83 & 47,95 & 24,40 & 1169,98 \\
\hline & Lona transparente & 1400(largura) & $\mathrm{m}^{2}$ & 6,83 & 7,52 & 14,17 & 106,56 \\
\hline & Chapa policarbonato compacto & $8 \times 3000 \times 2000$ & $\mathrm{~m}^{2}$ & 6,83 & 1,14 & 2550,00 & 2902,75 \\
\hline \multicolumn{8}{|l|}{ 3. ESTRUTURA } \\
\hline \multirow{7}{*}{$\begin{array}{l}\text { Função } \\
\text { Parede/ } \\
\text { Estrutural }\end{array}$} & $\begin{array}{l}\text { Divisória de parede madeira } \\
\text { plástica }\end{array}$ & $200 \times 70$ & $\mathrm{~m}$ & 47,02 & 172,95 & 38,00 & 6572,10 \\
\hline & Chapa de Polipropileno (PP) & $15 \times 2000 \times 1000$ & $\mathrm{~m}^{2}$ & 47,02 & 23,51 & 191,53 & 4502,87 \\
\hline & Chapa de PVC & $15 \times 1220 \times 2440$ & $\mathrm{~m}^{2}$ & 47,02 & 15,78 & 419,00 & 6611,20 \\
\hline & Perfil estrutural em alumínio 1 & $48,1 \times 24,4$ & $\mathrm{~m}$ & 40 & 6,67 & 5,34 & 35,60 \\
\hline & Perfil canaleta em alumínio 2 & $20 \times 20$ & $\mathrm{~m}$ & 4,7 & 0,78 & 2,46 & 1,93 \\
\hline & Caneleta PVC & $10 \times 20$ & $\mathrm{~m}$ & 44,2 & 22,1 & 3,2 & 70,72 \\
\hline & Luvas Alumínio & - & UM & - & 11 & 5,9 & 64,90 \\
\hline & & & & & & TOTAL & 7978,73 \\
\hline
\end{tabular}

Assim, de acordo com a tabela acima, é possível verificar que a quantidade de materiais necessários para a construção da nova proposta para o módulo, totalizam uma estimativa de preço de $R \$ 7.978,73$, para o novo módulo, sendo aproximadamente $R \$ 665,00 / \mathrm{m}^{2}$. Visto isso, abaixo seguem imagens da concepção do estudo efetuado, tal qual a explicação do detalhamento e da utilização dos elementos construtivos. Apresentam-se também, detalhes escolhidos para o novo sistema construtivo, assim como o empilhado do novo módulo. 
Figura 3 - Proposta de novo AEJ modelado no

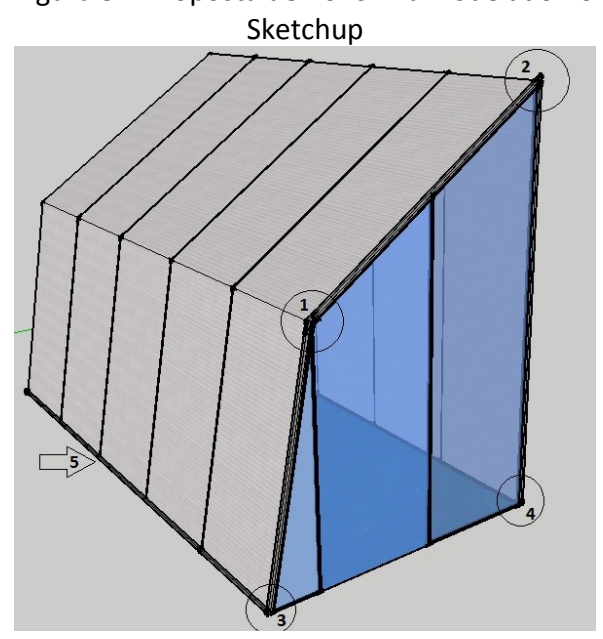

Figura 4 - Detalhamento 1, utilização de perfil canaleta de alumínio para travamento da estrutura

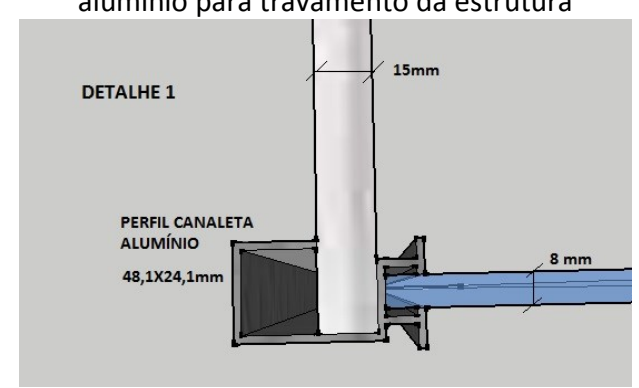

Fonte: Autora, 2016.

Fonte: Autora, 2016.

Figura 5 - Vista frontal da proposta do novo AEJ projetado no Sketchup

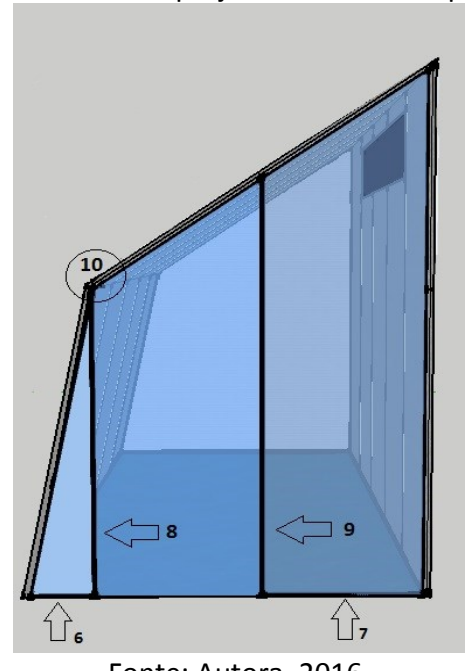

Figura 6 - Detalhe do perfil estrutural de alumínio DETALHE 8 e 9

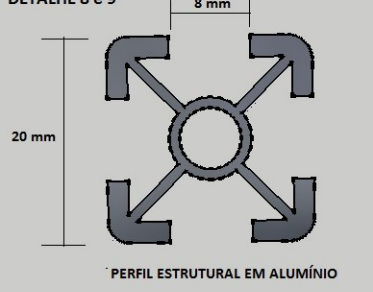

Fonte: Autora, 2016.

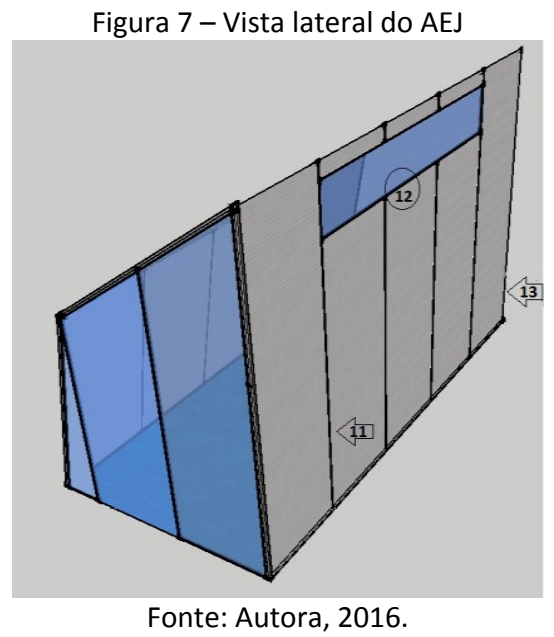

Sendo assim, os materiais selecionados para se consolidar o novo abrigo são: Lona Resistente, placa de policarbonato do projeto inicial e para o sistema da estrutura placas de polipropileno, e perfil de alumínio.

Sendo assim, as alterações que foram acatadas e utilizadas na concepção do novo projeto foram: $\mathrm{Na}$ infraestrutura propõe-se guia de alumínio para fixar a estrutura, além de encaixe por travas, ao fechamento sugere-se sistemas de encaixe e encaixe macho-fêmea e travas. Para a estrutura aplica-se chapas de polipropileno, além de terem sido descartadas as aberturas laterais em policarbonato, aumentado o tamanho da janela. 
existindo uma folga considerável nas medidas do container. Considerando que o novo projeto do Abrigo, possui a capacidade de abrigar 4 pessoas, e não mais 3, devido a mudanças realizadas no projeto da estrutura, os $30 \mathrm{KIT}$ 's teriam a capacidade de abrigar 120 pessoas. Na tabela 6 segue um resumo do comparativo do antigo módulo com o novo.

Tabela 4 - Comparativo do AEJ Original com a Nova Proposta para o AEJ

\begin{tabular}{|l|c|c|}
\hline \multicolumn{1}{|c|}{ CARACTERÍSTICAS } & AEJ ORIGINAL & NOVA PROPOSTA AEJ \\
\hline Dimensões do KIT $-\mathrm{c} \times \mathrm{I} \times \mathrm{h}(\mathrm{m})$ & $3,45 \times 2,20 \times 1,80$ & $3,40 \times 1,0,0,40$ \\
\hline Capacidade por módulo & 3 pessoas & 4 pessoas \\
\hline Quantidade de Módulos para a Situação 1 & 124 & 124 \\
\hline Capacidade de pessoas atendidas no estacionamento & 372 & 496 \\
\hline Capacidade de KITS por Caminhão Container & 3 & 5 \\
\hline $\begin{array}{l}\text { Quantidade de Caminhões Container para transporte } \\
\text { para Situação 1 }\end{array}$ & 41 & $\mathrm{R} \$ 7.978,73$ \\
\hline Custo de 1 Módulo (R\$) & $24.258,02$ & $989.362,50$ \\
\hline Custo Total (R\$) & $3.007 .994,50$ & \\
\hline
\end{tabular}

Portanto, para a nova proposta do Abrigo Emergencial para Joinville, constata-se que seria vantajoso optar pela troca de materiais e do sistema construtivo para viabilizar o projeto, reduzindo custos, garantindo a eficiência, qualidade final e a mantendo a segurança.

\section{CONCLUSÃO}

Os abrigos emergenciais são utilizados em situação adversa, principalmente ocasionada por desastres naturais. A importância de se fornecer abrigos para os atingidos, além da ideia de proteção e individualidade, é sua operação, que deve ser de rápido fornecimento, ter um baixo custo, ser executável, desmontável e adaptável a diferentes locais. Características essas que se encaixam em um Sistema Flat-Pack, que foi a base do trabalho.

Com o objetivo minimizar os custos, sem perder a identidade do projeto, um novo modelo foi estudado e apresentado, alterando elementos construtivos e materiais, no qual se alcançou um valor mais acessível, com maior capacidade de atendimento, redução dos tamanhos dos kits facilitando o transporte até o local. Cabe ressaltar que todo o desenvolvimento das adaptações do abrigo, foram feitas sob estimativas e baseadas em valores de mercado catarinense no ano de 2016.

\section{REFERÊNCIAS}

SINDUSCONBNU. Custo Unitário de Edificações Residenciais, Comerciais e Industriais. Disponível em: <http://sindusconpr.com.br/tabela-completa-370-p>. Acesso em: 16.mai.2016. 
